\title{
Reconstructing the Stellar UV and EUV Emission that Controls the Chemistry of Exoplanet Atmospheres
}

\author{
Jeffrey L. Linsky ${ }^{1}$, Kevin France ${ }^{2}$ and Tom Ayres ${ }^{2}$ \\ ${ }^{1}$ JILA, University of Colorado and NIST, Boulder CO 80309-0440, USA \\ email: jlinskyjila.colorado.edu \\ ${ }^{2}$ CASA, University of Colorado, Boulder CO 80309-0389, USA
}

\begin{abstract}
Lyman- $\alpha$ and extreme-ultraviolet radiation from exoplanet host stars are critically important for evaluating the phototchemistry of planetary atmospheres, but these emissions are largely or completely absorbed by hydrogen in the interstellar medium. We describe a new technique for estimating the intrinsic Lyman- $\alpha$ and EUV fluxes of F, G, K, and M stars using correlations with observable emission lines.
\end{abstract}

Keywords. exoplanet atmospheres, ultraviolet stellar spectra, extreme ultraviolet stellar spectra, hydrogen Lyman- $\alpha$ emission

\section{The Importance of Host Star Lyman- $\alpha$ and EUV Emission}

Since photodissociation and photoionization play critical roles in the chemistry of exoplanet atmospheres (e.g., Kopparapu, Kasting, \& Zahnle 2012 and Ribas et al. 2010), it is essential to identify the primary spectral features in a host star's spectrum and to measure the corresponding fluxes. This is not a simple task as interstellar neutral hydrogen obscures most of the flux in the important Lyman- $\alpha$ emission line and completely absorbs EUV radiation from $911 \AA$ down to at least $400 \AA$ even for very nearby stars.

Figure 1 shows the solar irradiance (flux of the entire Sun) at 1 AU. The near-ultraviolet (NUV, $\lambda=1700-3200 \AA$ ) radiation, produced in the stellar photosphere, is orders-ofmagnitude fainter in M dwarfs than for the Sun. However, the far-ultraviolet (FUV, $\lambda=$ 1170-1700 A) radiation, emitted by the stellar chromosphere, from GJ 876, a typical M5.0 V exoplanet host star, is similar to that of the Sun (in their corresponding habitable zones) (France et al. 2012). The 911-1170 $\AA$ spectral region containing the hydrogen Lyman series and other emission lines formed between 10,000 and 300,000 $\mathrm{K}$ is not observable by $H S T$, but was observed by the Far Ultraviolet Spectrograph Explorer (FUSE) satellite. The extreme-ultraviolet (EUV) spectrum at wavelengths shorter than the hydrogen ionization edge $(911 \AA)$ shown in Figure 2 consists of hydrogen and helium continua and emission lines formed in the chromosphere, transition region, and corona (mostly shorter than $400 \AA$ ).

Host star FUV radiation photodissociates many important molecules, e.g., $\mathrm{H}_{2} \mathrm{O}, \mathrm{CO}_{2}$, $\mathrm{CH}_{4}, \mathrm{C}_{2} \mathrm{H}_{2}, \mathrm{C}_{2} \mathrm{H}_{6}$, in the atmospheres of exoplanets. Figure 3 shows the cumulative contribution of the solar FUV spectrum times the $\mathrm{H}_{2} \mathrm{O}$ photodissociation cross-section. Note that the solar Lyman- $\alpha$ line is responsible for fully half of the photodissociation rate of $\mathrm{H}_{2} \mathrm{O}$. Since for $\mathrm{M}$ dwarfs the NUV flux is far smaller than for the Sun, while the Lyman- $\alpha$ line is similar to that of the Sun (see below), the Lyman- $\alpha$ line in M dwarfs is responsible for essentially the entire photodissociation rate for this important molecule. Figure 4 shows that the solar Lyman- $\alpha$ line dominates the photodissociation rate for 


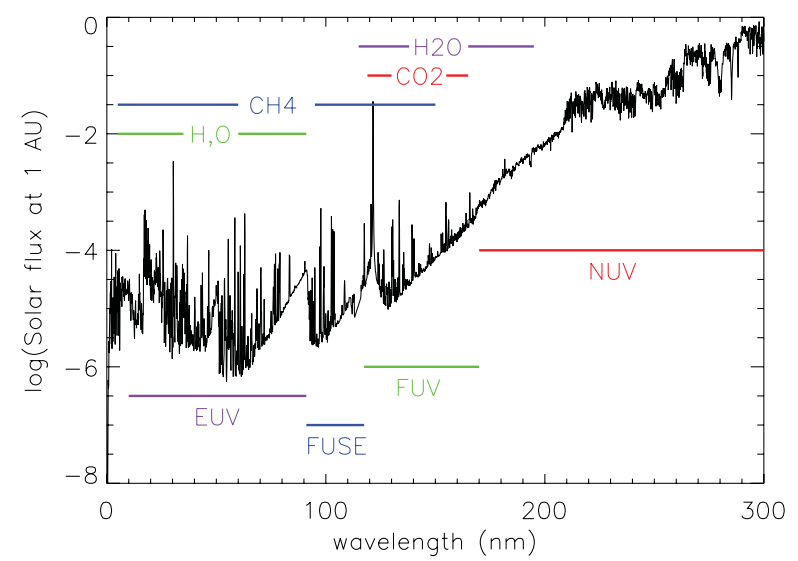

Figure 1. The quiet Sun irradiance spectrum observed on April 10-16, 2008, with the Solar Radiation and Climate Experiment (SORCE) on the SOLSTICE II satellite (Snow et al. 2005, Woods et al. 2009). Different spectral ranges are indicated as well as the overlap with the photodissociation and photoionization cross-sections of the $\mathrm{H}_{2} \mathrm{O}, \mathrm{CO}_{2}$, and $\mathrm{CH}_{4}$ molecules and hydrogen and oxygen atoms.

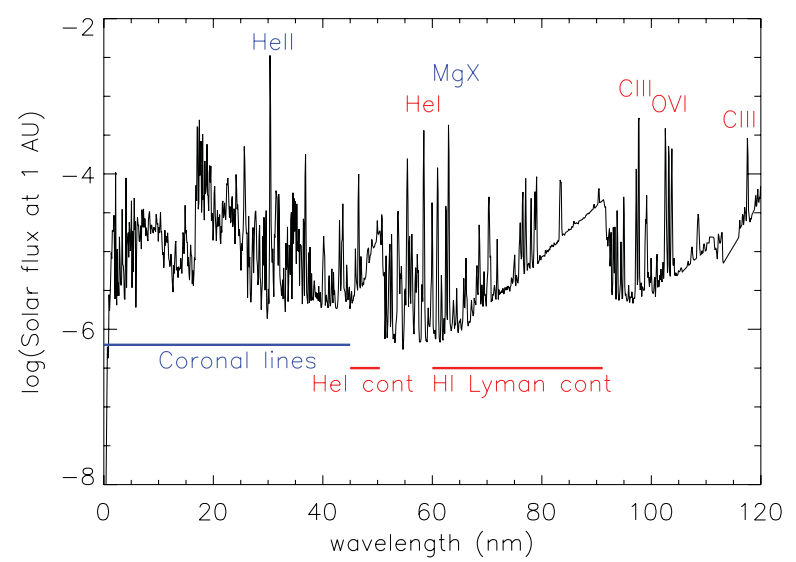

Figure 2. The EUV portion of the quiet Sun irradiance spectrum showing the presence of the hydrogen Lyman continuum and neutral helium continuum. In this spectral region, there are many lines formed in the chromosphere (e.g., the hydrogen Lyman series and C II) and the transition region (e.g., C III, C IV, and O VI). Below $40 \mathrm{~nm}$, most emission lines are formed in the corona. The bright He II Lyman- $\alpha$ line at $30.4 \mathrm{~nm}$ is formed partially by electron collisions in the chromosphere and partially by recombination following X-ray ionization.

methane with a small contribution by the EUV flux. EUV radiation photoionizes and heats important atoms including hydrogen and oxygen in planetary exospheres (MurrayClay, Chiang, \& Murray, N. 2009).

\section{Reconstructing the Stellar Lyman- $\alpha$ and EUV Flux}

There are three techniques for reconstructing or estimating the intrinsic Lyman- $\alpha$ emission line. If one has a high-resolution Lyman- $\alpha$ spectrum (typically with the STIS instrument on $H S T$ ) and knowledge of the interstellar hydrogen-gas velocity structure and column density along the line of sight (e.g., Redfield \& Linsky 2008), then one can correct for the interstellar absorption to obtain the intrinsic Lyman- $\alpha$ emission line and 


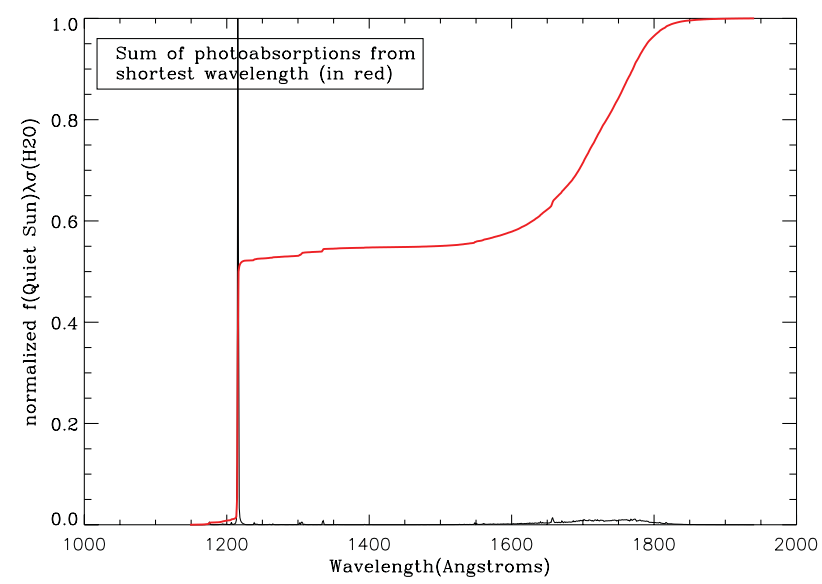

Figure 3. Overlap of the solar quiet Sun irradiance with the $\mathrm{H}_{2} \mathrm{O}$ photodissociation cross-section. The plot shows the sum of the overlap integral starting with the shortest wavelengths. Lyman- $\alpha$ emission is responsible for about half of the dissociation rate for solar-type stars and far more for cooler stars.

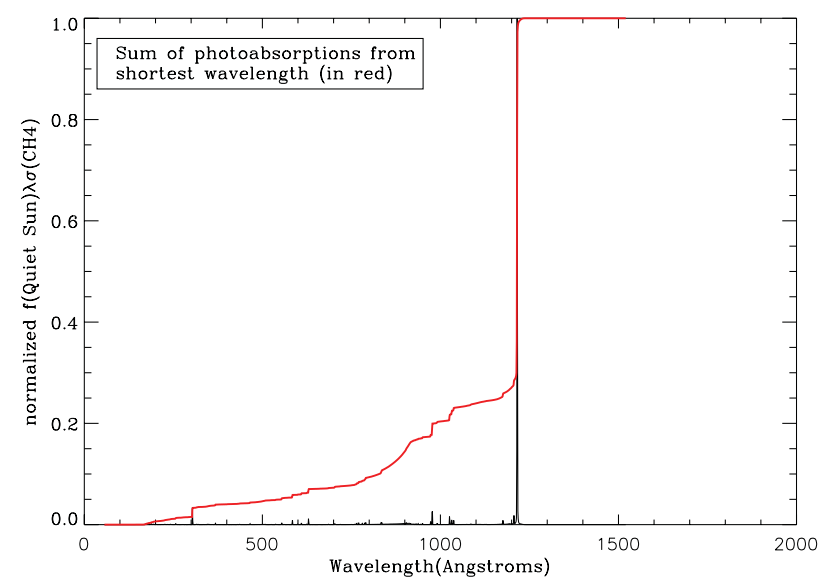

Figure 4. Same as Figure 3 except for the $\mathrm{CH}_{4}$ molecule. Lyman- $\alpha$ emission is responsible for more than $70 \%$ of the photodissociation rate.

flux. Wood et al. (2005) has done this for 62 stars (including 42 main sequence stars), most of which are located within $50 \mathrm{pc}$ of the Sun. If instead one has a high-resolution Lyman$\alpha$ spectrum but no a priori information on the interstellar medium along the line of sight, then one may be able to solve iteratively for both the width of the intrinsic Lyman- $\alpha$ line and the interstellar absorption, as shown by France et al. (2012). We include in our study the reconstructed Lyman- $\alpha$ fluxes for five M dwarf host stars (GJ 832, GJ 667C, GJ 876, GJ 581, and GJ 436) computed with this technique (France et al. 2013).

With reconstructed Lyman- $\alpha$ fluxes of 47 main-sequence stars available for use with the first two reconstruction techniques, we consider whether there are any useful trends in these fluxes with stellar effective temperature and chromospheric activity. Ribas et al. (2005) have previously shown that broadband X-ray, EUV, and UV fluxes for solartype stars depend on stellar age. In Figure 5, we plot the Lyman- $\alpha$ flux in a star's habitable zone (scaled according to the stellar lumnosity) as a function of stellar effective temperature, $T_{\text {eff }}$. There is a factor of 6-30 dispersion in Lyman- $\alpha$ flux at a given $T_{\text {eff }}$. This large range is reduced by grouping stars according to rotational period, which is a 


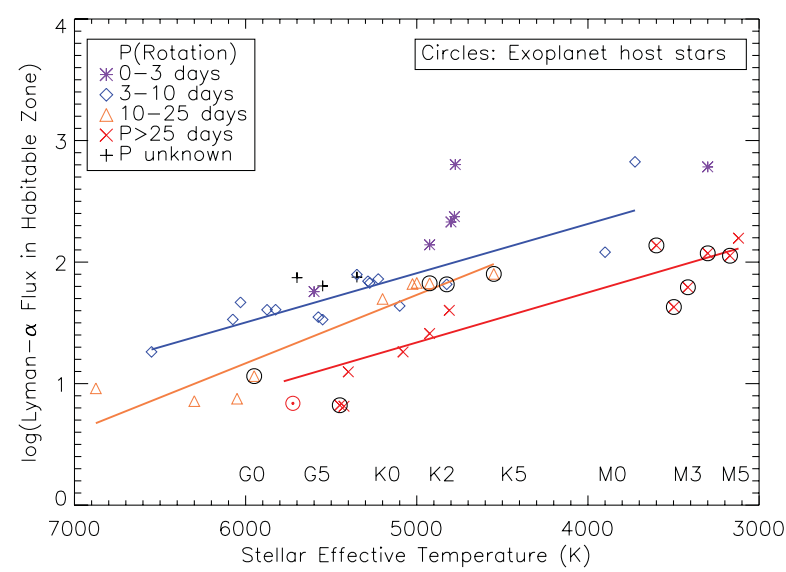

Figure 5. Comparison of the reconstructed Lyman- $\alpha$ flux in a star's habitable zone vs. stellar effective temperature for the 47 main sequence stars with available data. Different symbols and colors identify stellar rotational periods indicative of magnetic activity and heating. Exoplanet host stars are circled, and the quiet and active Sun data are identified by dotted-circle symbols. Solid lines are least-squares fits to stars with similar rotational periods. Note the large range in Lyman- $\alpha$ flux for stars of similar effective temperatures, and that M dwarfs have larger Lyman- $\alpha$ fluxes in their habitable zones than the Sun at $1 \mathrm{AU}$.

measure of magnetic activity and heating. The dispersion of Lyman- $\alpha$ flux about leastsquares fits to the data for stars of similar rotational period is relatively small for stars with $T_{\text {eff }}>5000 \mathrm{~K}$, but becomes large for the cooler stars. If the rotational period of a star is known, even roughly, the least-squares fits shown in Figure 5 should provide good estimates of the intrinsic Lyman- $\alpha$ flux for the warmer stars.

For many important exoplanet host stars, there are no available Lyman- $\alpha$ data to reconstruct. For these stars, Linsky, France, \& Ayres (2012) developed a new approach for estimating the intrinsic Lyman- $\alpha$ flux from correlations with other emission lines formed in the chromosphere and transition region. Fontenla et al. (2011) and previously Avrett \& Loeser (2008) computed semiempirical models of solar atmosphere regions with different amounts of magnetic heating. These models have temperature vs. height structures that are very similar in shape, but displaced deeper into the atmosphere to higher densities as the heating rate increases. This displacement suggests that flux ratios of emission lines formed at similar, and perhaps even very different, temperatures should be roughly constant or change slowly with emission line flux.

To test this hypothesis, Linsky et al. (2012) plotted the ratios of reconstructed Lyman$\alpha$ line flux to the emission lines of Mg II, O I, C II, and C IV, which can be observed with spectrometers on HST. Figure 6 is an example of such a plot using the C IV emission lines. For stars in different spectral class groups, there is a relatively small scatter (typically $20 \%$ ) of the Lyman- $\alpha /$ C IV ratio vs. C IV irradiance (at a standard distance of 1 AU) about the least-squares fit to all of the data for each spectral type group. The slopes of the fit lines are similar except for the M stars. In many cases, the stars with low or suspected low-metal abundances (marked L and $\mathrm{H}$ ) lie above the fit lines, as would be expected for stars with low carbon abundance. This plot, and similar correlation plots with Mg II, O I, and C II, show that one can estimate the Lyman- $\alpha$ flux of a star with no Lyman- $\alpha$ spectrum, provided one has a measurement of one of the other emission lines. If the star has abundances similar to the Sun, this method should provide Lyman- $\alpha$ fluxes with an uncertainty of about $20 \%$. 


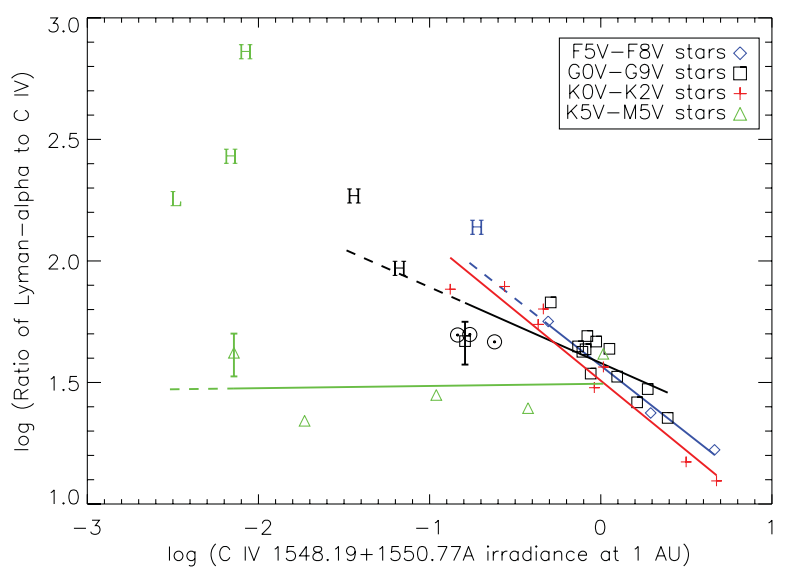

Figure 6. Plot of the ratio of the reconstructed Lyman- $\alpha$ flux to the C IV $1548+1550 \AA$ flux vs. the C IV flux at 1 AU. Stars in different spectral type groups are identified by symbol types and colors. Dotted circles identify quiet and active Sun data, H symbols identify high proper motion stars, which likely have low metal abundances, and L symbols identify low-metal abundance stars. Solid lines are least-squares fits to the data for each spectral type group, except for the $\mathrm{H}$ and $\mathrm{L}$ stars. Note that the scatter about the least-square lines is typically less than $20 \%$, indicating that the least-squares fit lines provide good estimates of the Lyman- $\alpha$ flux for stars that have near-solar metal abundances.

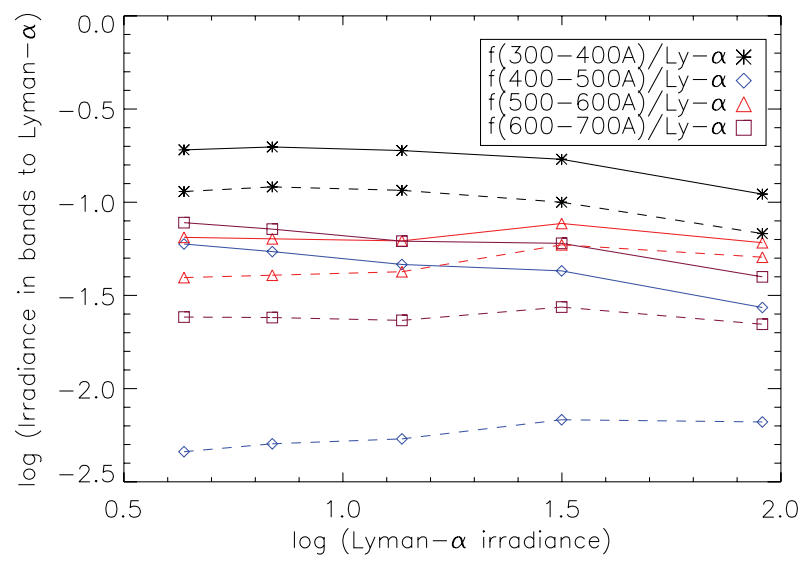

Figure 7. Plots of the solar EUV irradiance in $100 \AA$ bands to Lyman- $\alpha$ vs. the Lyman- $\alpha$ irradiance for five semiempirical chromosphere and corona models computed by Fontenla et al. (2011) to match the spectra of regions of the Sun with faint- (left) to strong- (right) UV emission. The solid lines refer to the combined emission of the corresponding chromosphere and corona models, and the dashed lines refer to emission only from the chromosphere models. Note that the EUV irradiance is proportional to the Lyman- $\alpha$ irradiance.

Linsky et al. (2012) extended this correlation method to estimate fluxes in the EUV. Figures 7 and 8 show ratios of the EUV flux in $100 \AA$ bands to Lyman- $\alpha$ using the Fontenla et al. (2011) models, which were constructed to fit UV and EUV fluxes in solar regions with a range of 30 in Lyman- $\alpha$ flux. With one exception, the plots are horizontal, indicating that the EUV flux in these bands is directly proportional to the Lyman- $\alpha$ flux. While semiempirical models of this quality for $\mathrm{K}$ and $\mathrm{M}$ dwarfs are not yet available, we anticipate that the EUV flux for these stars can also be estimated from the Lyman- $\alpha$ flux, which can be estimated from correlations with other emission lines. 


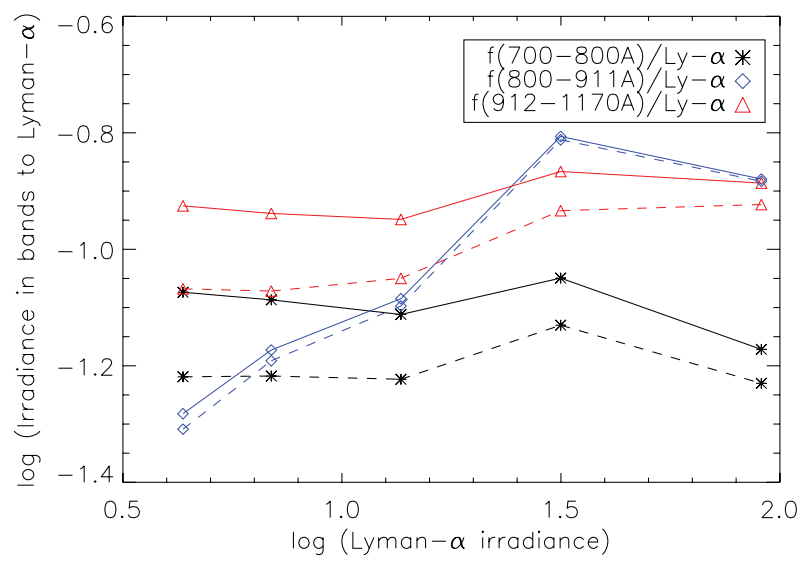

Figure 8. Same as Figure 7 except for EUV bands at longer wavelengths.

The authors are Guest Observers, NASA/ESA Hubble Space Telescope and Users of the Data Archive of the Space Telescope Science Institute. STScI is operated by AURA, Inc., under NASA contract NAS5-26555. This work is supported by NASA through grants NNX08AC146, NAS5-98043, and HST GO-11687.01A to the University of Colorado at Boulder. We thank Martin Snow for providing the SORCE data.

\section{References}

Avrett, E. H. \& Loeser, R. 2008, ApJS, 175, 229

Fontenla, J. M., Harder, J., Livingston, W., Snow, M., \& Woods, T. 2011, JGR, 116, D20108

France, K., Linsky, J. L., Feng, T., Froning, C. S., \& Roberge, A. 2012, ApJ, 750, 32

France, K., et al. 2013, ApJ, 763, article id.149

Kopparapu, R. K., Kasting, J. E., \& Zahnle, K. J. 2012, ApJ, 745, 77

Linsky, J. L., France, K, \& Ayres, T. 2013, ApJ, 766, article id.69

Murray-Clay, R. A., Chiang, E. I., \& Murray, N. 2009 ApJ, 693, 23

Ribas, I., Guinan, E. F., Güdel, M., \& Audard, M. 2005, ApJ, 622, 680

Ribas, I., Porto de Mello, G. F., Ferreira, L. D., Hébrard, E., Selsis, F., Catalán, S., Garcés, A., do Nascimento Jr., J. D., \& de Medeiros, J. R. 2010, ApJ, 714, 384

Snow, M., McClintock, W. E., Rottman, G., \& Woods, T. N. 2005, Solar Phys., 230, 295

Wood, B. E., Redfield, S., Linsky, J. L., Müller, H.-R., \& Zank, G. P. 2005, ApJS, 159, 118

Woods, T. N., et al. 2009, J. Geophys. Res., 36, L01101 